\title{
Ultra Wide Band Pulse Generation Using Advanced Design System Software
}

\author{
Mr. Anish Dhabliya \\ Developer, AMDOCS Pune India \\ anishdhablia@gmail.com
}

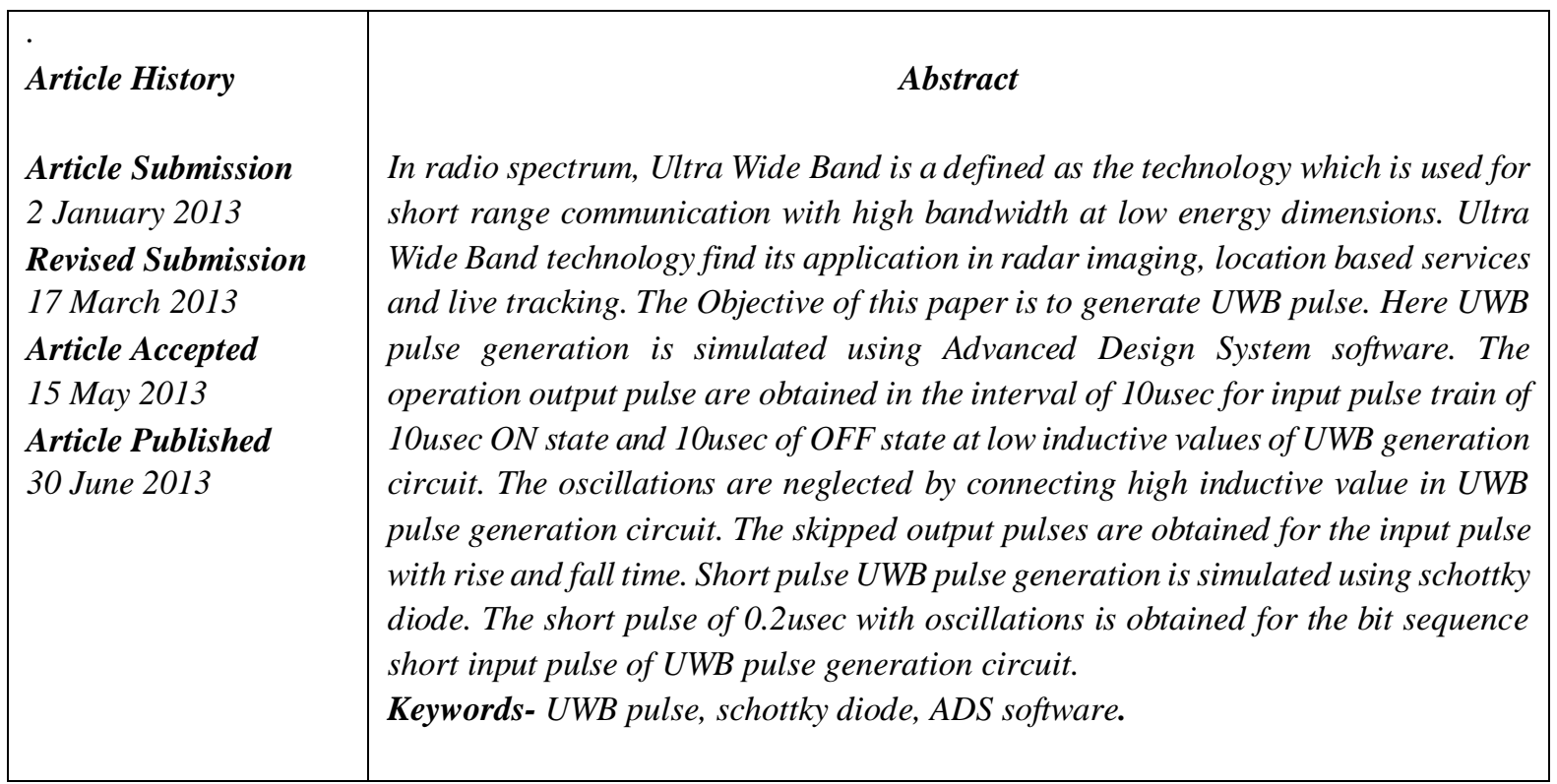

\section{Introduction}

The generation and transmission of the UWB pulse in the range of $3 \mathrm{GHz}$ to $10 \mathrm{GHz}$ for the detection and investigate the information of the object behind the wall [1][2]. UWB pulse has the property of both penetrating and reflecting when it hit an object. The object can be either a living or non-living. But the signal varies for the living and non-living object [3][5]. The living object produces the variations in the received signal data due to their frequent movement, physical activity, inhalation and heart beat [6]. The non- living objects produce the variations, if it is being moved and sometimes due to the periodic functions such as the rotator function in case of a fan or a motor. The advantage of the UWB signal is the short pulse width which might have radar resolution benefits [4].

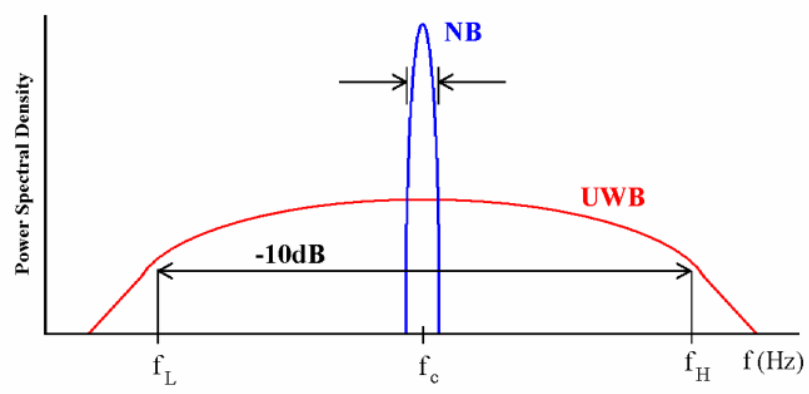

Fig. 1: An UWB Pulse Signal

Commonly used signals for UWB applications are step-like pulse, impulse, rectangular pulse, mono cycle and poly cycle pulse. To radiate at the frequency between $3.1 \mathrm{GHz}$ to $10.6 \mathrm{GHz}$, these signals must be shaped with some kind of filtering techniques. Therefore, it is important to understand the time transient analysis response and 
frequency spectral content of these signals [7]. The ITU describes the Ultra wideband signal as pulse radio whose signal bandwidth exceeds $500 \mathrm{MHz}$ or $20 \%$ of the provided center frequency. Under FCC regulation, the UWB is defined by its operating frequency and the occupied fractional bandwidth [8]. The signals lie between $3.1 \pm$ $10.6 \mathrm{GHz}$ fractional bandwidth is larger than 0.2 [9][10].

Where fractional bandwidth

$$
F B=2 \times\left[f_{H} \pm f_{L}\right] /\left[f_{H}+f_{L}\right]
$$

Where fH - Upper cut off frequency with -10db emission point

fL - Lower cut off frequency with -10db emission point

\section{Proposed UWB Pulse Generation Method}

The generation of UWB signal requires a massive bandwidth efficient equipment whereas the available laboratory signal producers are capable of generating signals in terms of $10 \mathrm{MHz}$ to $100 \mathrm{MHz}$. The simple representation of UWB signal generation is shown in figure 2.

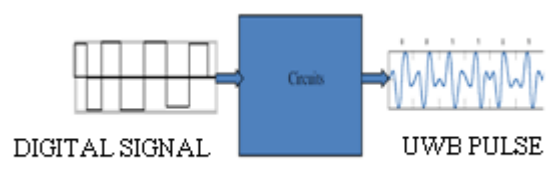

Fig.2: Generalized schematic for generation of UWB pulses.

Normally UWB signals are generated through modulators comprising of pulse generator, impulse generator, pulse shaping circuits and reconstruction filter. The generation of UWB signal requires baseband bandwidth in the range of GHZ. This drawback is overcome in the proposed method by introducing up conversion method. The block diagram shows the UWB pulse generation process in figure 3 .

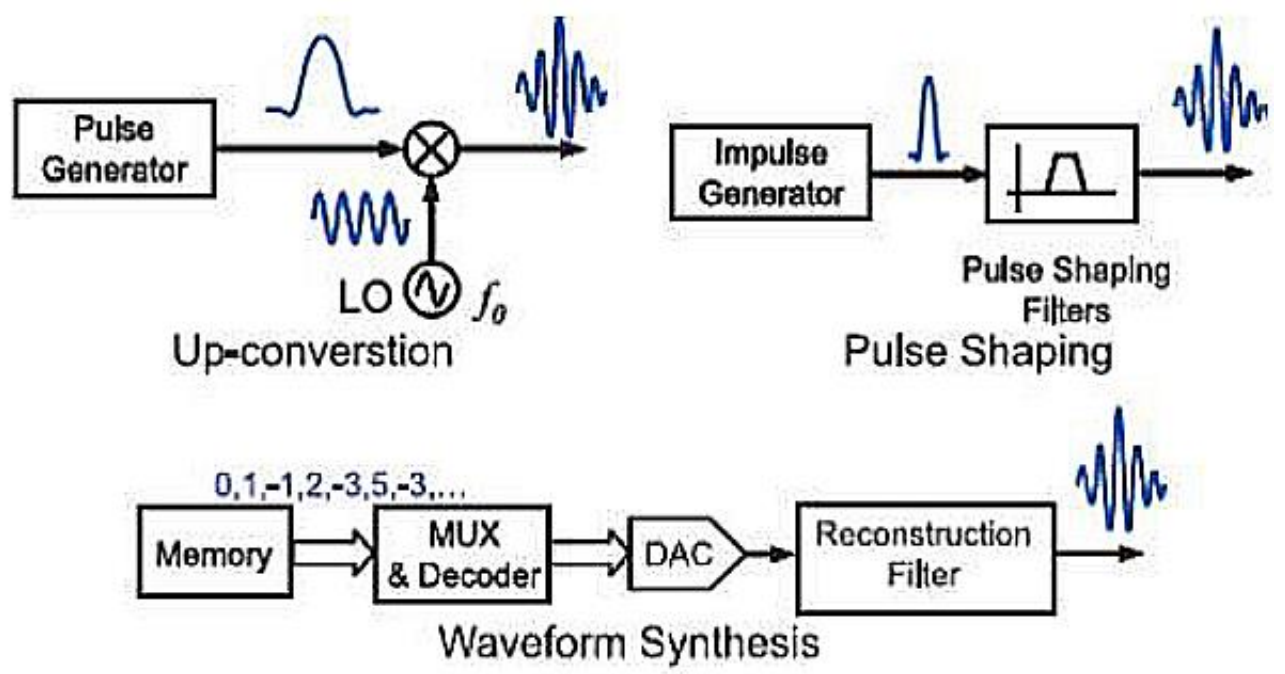

Fig. 3: UWB Pulse Generation Block Diagram

The circuit level implementation of UWB pulse generator is shown in fig.4. The circuit is tested at low frequencies and explained in the simulation section which is a HEMT based circuit. The inductor $\mathrm{L}$ and capacitor $\mathrm{C}$ values to be connected to the drain gets $800 \mathrm{nH}$ and $1 \mathrm{nF}$ respectively. The input pulse train to the circuit comprises of $\mathrm{V}_{\text {low }}$ $=2 \mathrm{~V}$ to $5 \mathrm{~V}, \mathrm{~V}_{\text {high }}=0 \mathrm{~V}$ to $15 \mathrm{~V}$ with a pulse rate of $5 \mathrm{kHz}$. Rise time and fall time have are maintained at $100 \mathrm{~ns}$. 


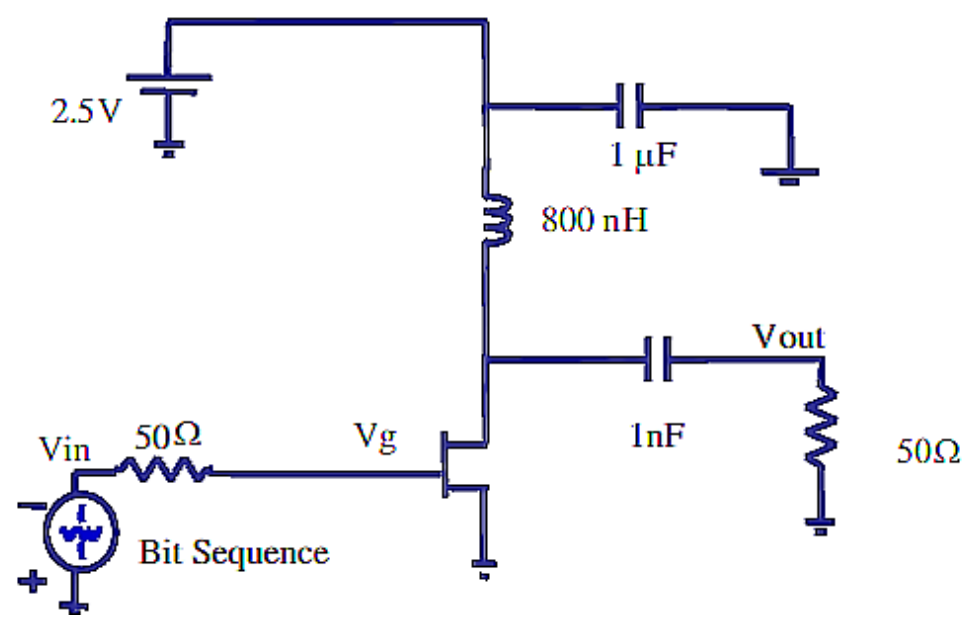

Fig-4 UWB pulse generation circuit at low frequency

The modified UWB pulse generation circuit is shown in Fig.5. A scotty diode shunted with a resistor and capacitor is added up at the input of the transistor. When a low level input signal is given as input, the FET transistor is turned off leading to the flow of drain current to LC circuit, thereby creating an UWB pulse. When a high level input signal is given as input, the FET transistor is turned on leading to the generation of a weak pulse.

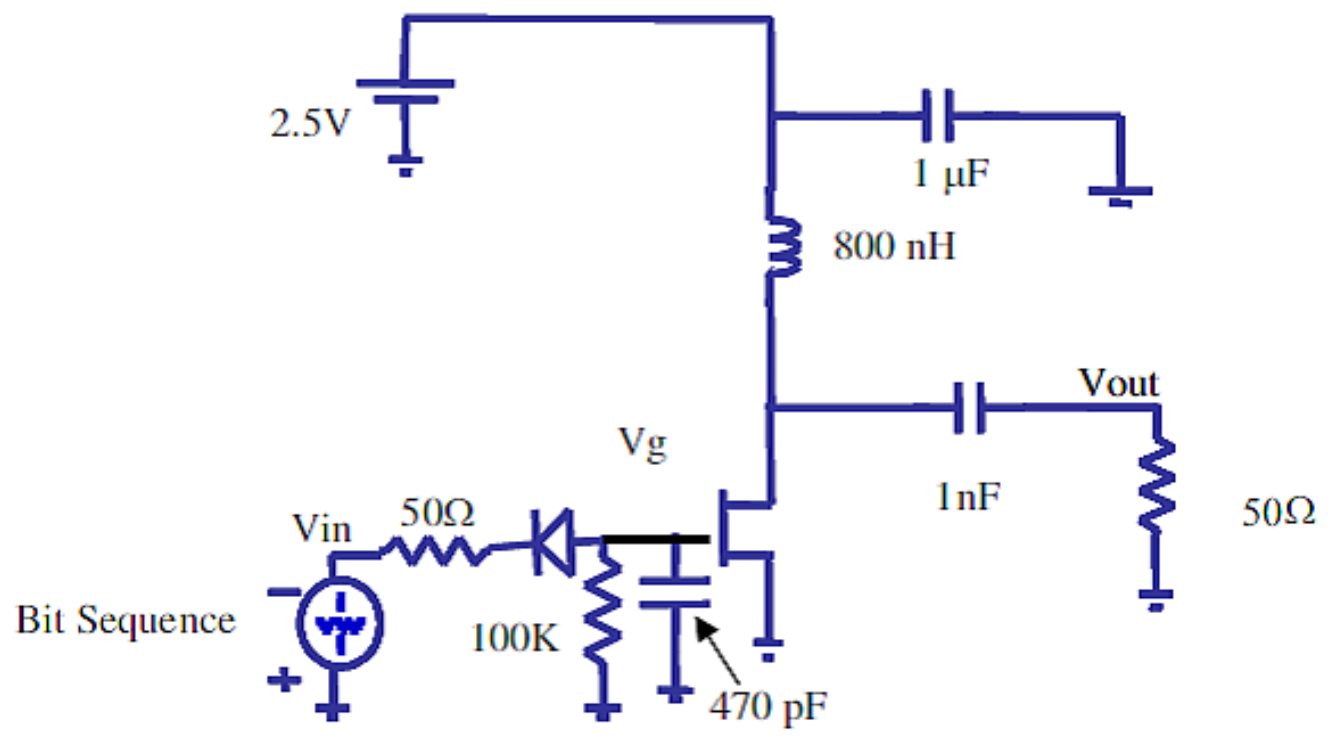

Fig-5 Modified Short pulse generation at low frequency

\section{Simulation Results}

Generation of UWB signal pulse have been simulated by Advanced Design System (ADS). UWB signals are normally generated through differentiator circuits followed by up converters. The other method employs the frequency spectrum provided by FCC regulation. The simulated circuit of approach 1 is shown in figure 6 . 


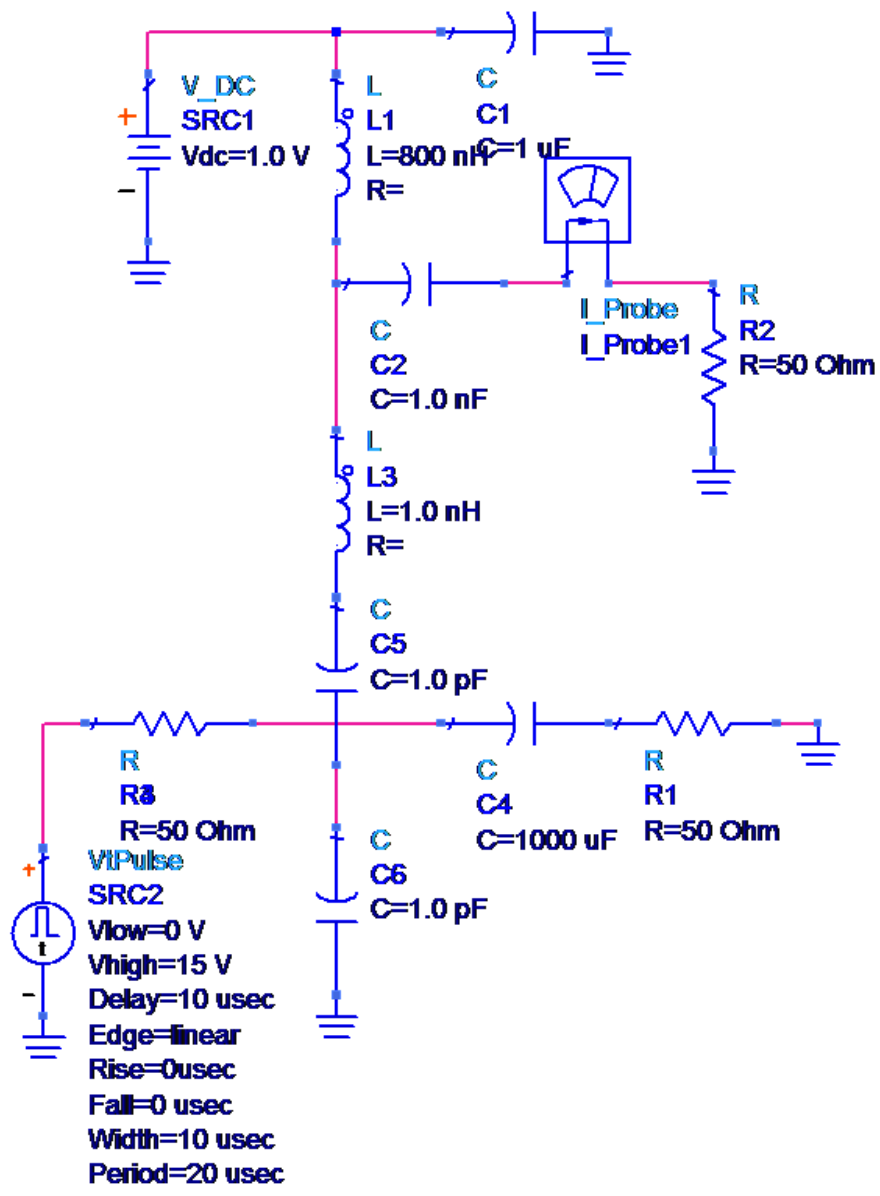

Fig-6 Simulation Circuit for UWB pulse generation

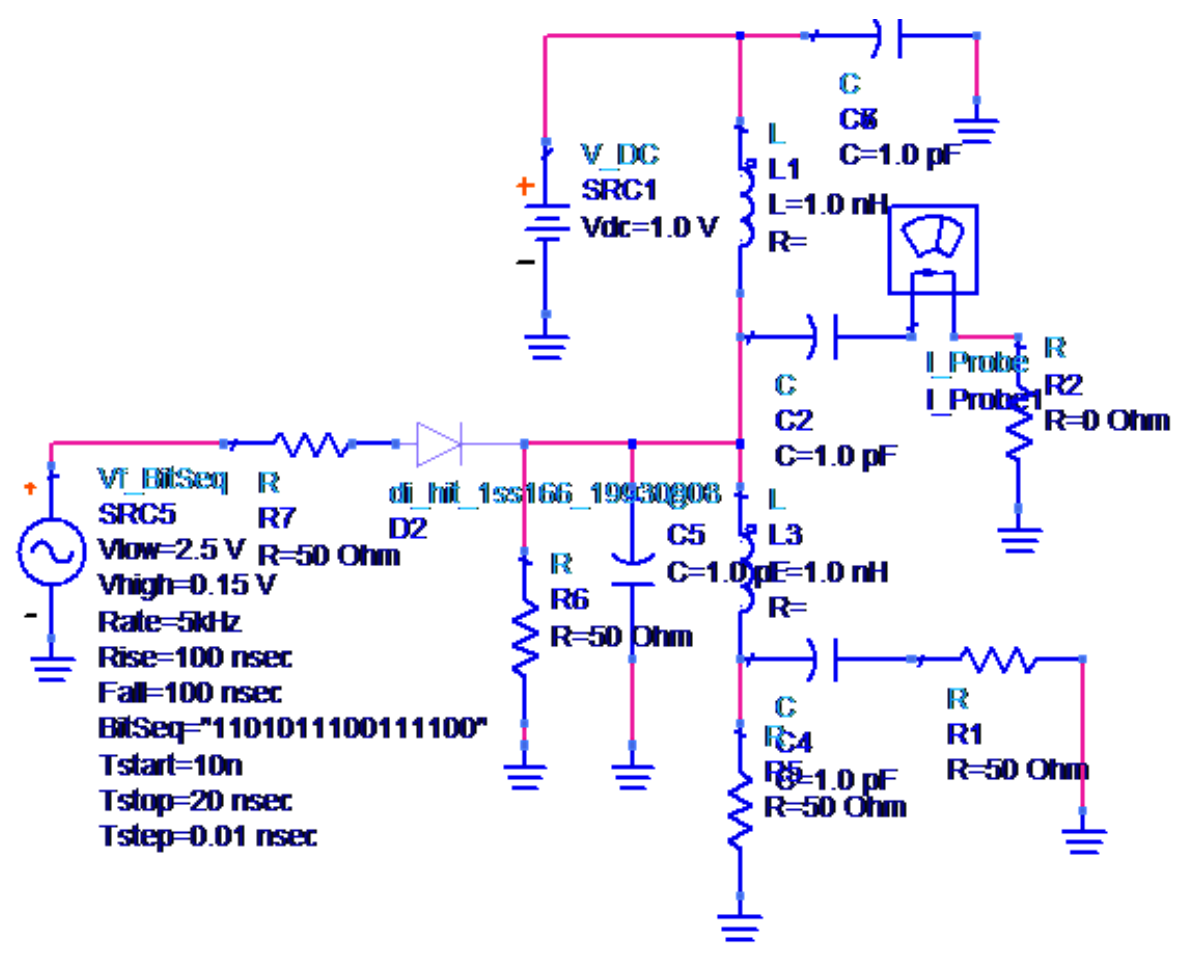

Fig-7 Simulation circuit of generation of short pulse with modified circuit 
A new scheme is proposed for the generation of short pulse using a FET where the modified pulse generation circuit is employed is shown in figure 7. In fig.7, Bit sequence is given as input pulse. Schottky diode is used for blocking AC voltage and to allow the dc voltage. Due to low resistance value and the high inductance value the bunched output voltage is obtained.

Fig-8 Shows input and output of UWB pulse generation with low inductive values. The graph is plotted for output voltage Vs time oscillated output is obtained because of low inductance value. The oscillated output pulses are obtained in the interval of 10usec for the input pulse train of 10usec ON state and 10usec OFF state.

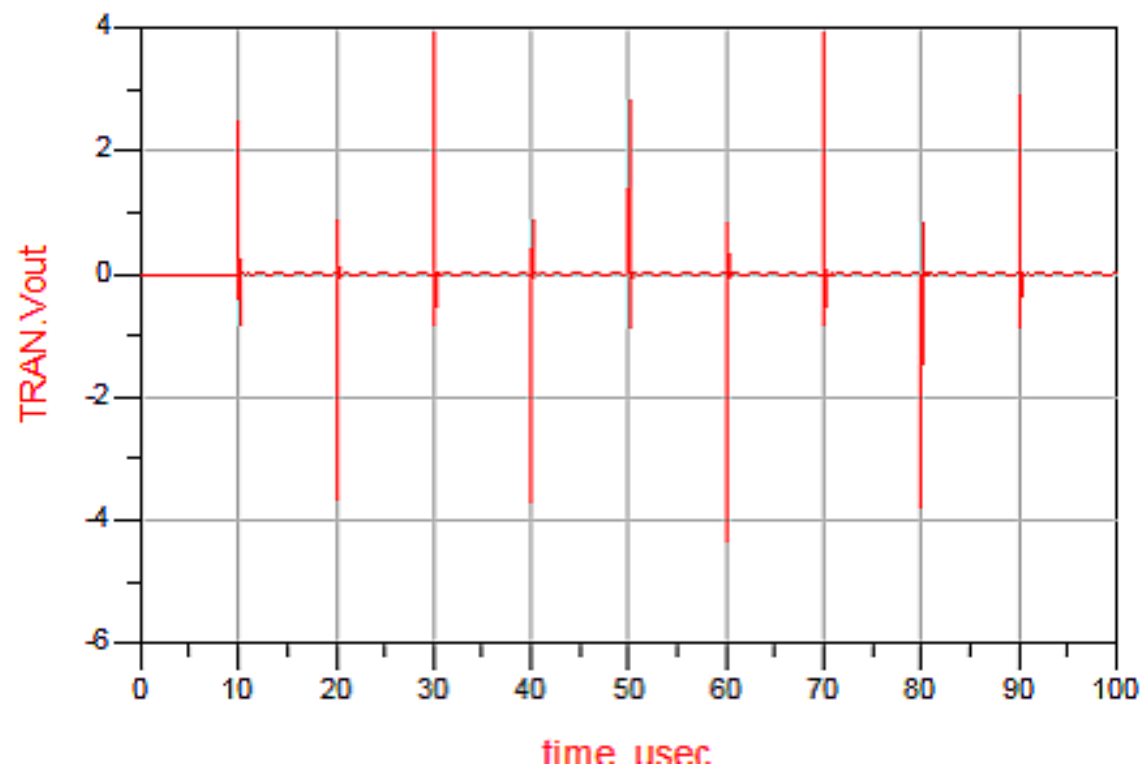

Fig-8 Output UWB pulse with low inductive value

The same input pulse that is given to the circuit in the simulator and the corresponding simulated output pulses are shown in Fig-8. Figure 9 shows the skipped output for the given input pulse with rise and fall time of 10usec. The skipped voltage varies with time.

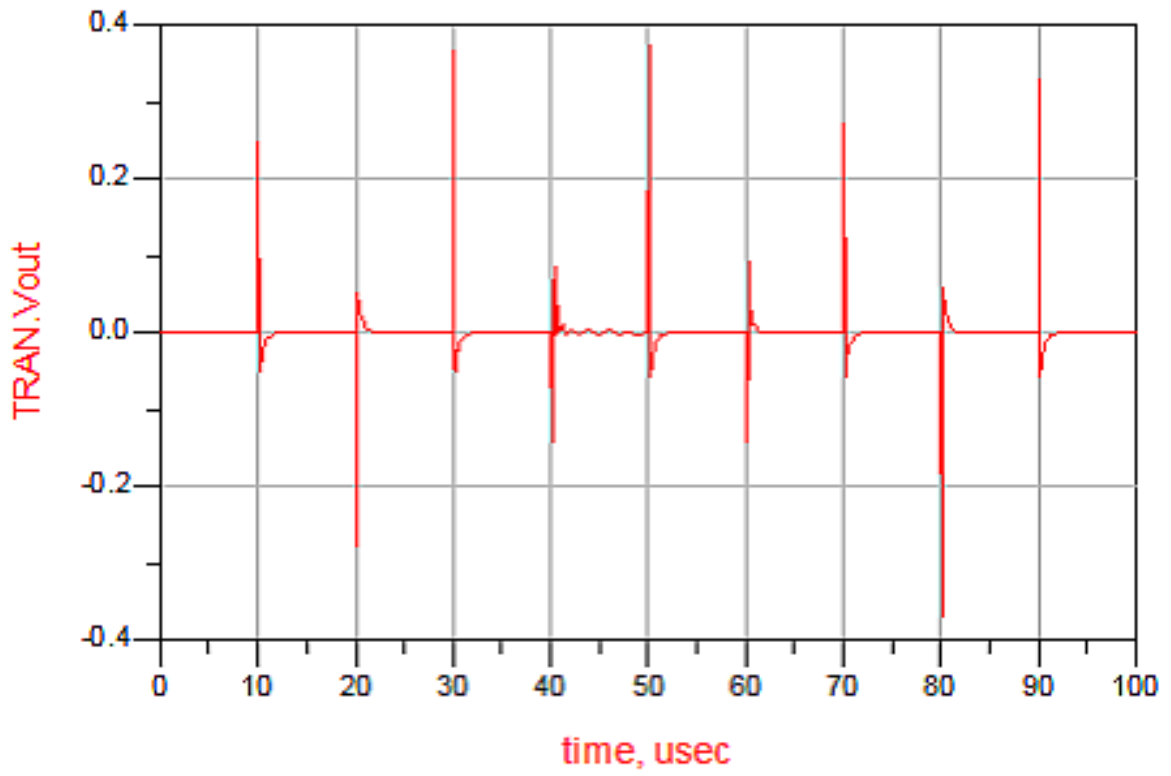

Fig. 9: Output signal of UWB pulse generation with rise and fall time 


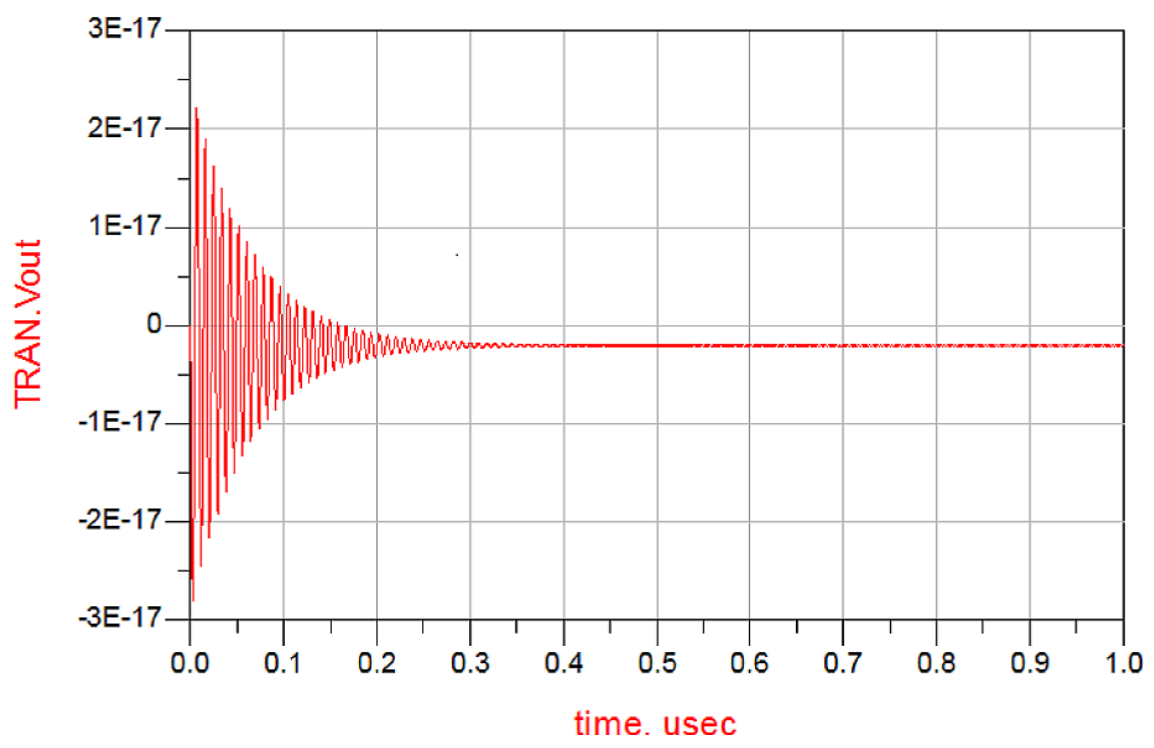

Fig.10: Simulation output for short pulse UWB generation

Fig-10 shows plot for output voltage Vs time where the maximum output is obtained at $2 \mathrm{E}-17$ and $-2 \mathrm{E}-17$. In the range of 0 to 0.2 oscillations are more and after 0.2 the constant output is obtained. The value of resistance $\mathrm{R}$ and capacitance $\mathrm{C}$ is determined by the switching mechanism.

\section{Conclusion}

In this paper, simplest circuits have been designed and simulated for generation of UWB pulse. This simulation has been carried out using Advanced Design System software. This UWB pulses can be able to penetrate through the barriers like wall and used to investigate the object behind the wall. This finds main application in military and radar, wireless communication. For the given input train pulse of 10us, the operating output pulses has been obtained at the interval of $10 \mathrm{usec}$. The skipped output pulses have been obtained for the input pulse of rise and fall time. The short pulse of $0.2 \mathrm{usec}$ with oscillations has been obtained for the bit sequence input pulse.

\section{References}

[1] Nadia Maaref, Patrick Millot, Christian Pichot(2010) 'Ultra Wide Band RADAR System for ThroughThe-Wall Microwave Localization and Imaging',IEEE CONFERENCE PAPER (EUSAR 2010).

[2] M.Kumar, A.Basu and S.K.Koul, 'Circuit in active Antennas for ultra wide band pulse generation and transmission', Progress in electromagnetic research B,Vol.23, 251-272,2010.

[3] Shravan Shirodkar, paramita Barua, Anuradha D and R.Kuloor (2011), 'Heart beat detection and ranging through a wall using Ultra Wide Band RADAR', www.ieeexplore.org.

[4] Nadia Maaref, Patrick Millot, Christian Pichot (2009), 'A study of Ultra Wide Band FMCW RADAR for detection of human beings in motion inside a building', IEEE Transaction Geoscience and Remote Sensing, Vol.47, No.5

[5] A.n.Gaikwad, P.K. Verma, D. Singh, and M.J. Nigam(2009), 'Analysis of clutter reduction techniques for through wall imaging in UWB range', Progress In Electromagnetics Research B, Vol.17.29-48

[6] Gregory L. Charvat, and Leo C. Kempel(2004), 'Low-Cost, High Resolation X-Band Laboratory RADAR System for Synthetic Aperature RADAR Applications', IEEE Tanscations, Antenna and Propagations., Vol.47, No.1

[7] E.Aggelopulos, E.karabetsos, P.Constantinou, N.Uzunoglu(2008), 'Microwave System Detection of Trapped Human Beings', IEEE Transcations, Antenna and Propagation., Vol.49,No.8 
[8] J. Yang (2013), "Calculation of the phase center of an ultra-wideband feed for reflector antennas," 2013 Proceedings of the International Symposium on Antennas \& Propagation, Nanjing, pp. 30-32.

[9] S. W. Wong, W. Liao, K. Wang and Q. Chu (2013), "Ultra-wideband (UWB) bandpass filter with three transmission zeros in the notched band," 2013 IEEE International Wireless Symposium(IWS), Beijing, pp. 1-4.

[10] T. Jaeschke, C. Bredendiek and N. Pohl (2013), "A 240 GHz ultra-wideband FMCW radar system with on-chip antennas for high resolution radar imaging," 2013 IEEE MTT-S International Microwave Symposium Digest (MTT), Seattle, WA, pp. 1-4. 